\title{
AUTOEVALUACIÓN Y RENOVACIÓN DE LA ACREDITACIÓN DEL PROGRAMA DE ODONTOLOGÍA DE LA UNIVERSIDAD SANTO TOMÁS DE BUCARAMANGA
}

\author{
María Claudia Latorre Gómez \\ Odontóloga U. Santo Tomás, Magíster en Evaluación en Educación U. Santo Tomás, \\ Coordinadora de Autoevaluación y Regulación de la Facultad de Odontología, \\ Coordinadora de Autoevaluación y Regulación Académica CEPA, U. Santo Tomás Seccional Bucaramanga, Docente U. Santo Tomás
}

Dirección de correo electrónico: mclatorre12@hotmail.com

\section{RESUMEN}

La Universidad Santo Tomás Seccional Bucaramanga ha mantenido y proyectado un compromiso con los procesos de alta calidad en sus programas reconocido por la comunidad académica a través de pares externos y por el gobierno nacional, que obedece al desarrollo de los ejes estratégicos definidos en su Plan de Acción articulado con la autorregulación de la institución y de sus programas de pregrado y posgrados. ${ }^{1}$ El presente artículo pretende registrar el proceso de autoevaluación y de mejoramiento continuo desarrollado por la Facultad de Odontología de la Universidad Santo Tomás de Bucaramanga durante la vigencia de la acreditación de alta calidad otorgada por el Ministerio de Educación Nacional por Resolución 3164 del 11 de diciembre del año 2003. El reconocimiento de alto nivel de calidad renovado mediante la Resolución 517 del 14 de febrero de 2008, se convierte en estímulo para el mejoramiento continuo en respuesta a las necesidades locales, regionales, nacionales e internacionales. [Latorre MC. Autoevaluación y renovación de la acreditación del programa de odontología de la Universidad Santo Tomás de Bucaramanga. Ustasalud Odontología 2008; 7: 12 - 18]

Palabras clave: Autoevaluación, Autorregulación, Acreditación.

\section{SELF EVALUATION AND RENEWAL OF THE DENTISTRY PROGRAM ACCREDITATION OF SANTO TOMAS UNIVERSITY BUCARAMANGA}

\section{ABSTRACT}

The Santo Tomas University Campus Bucaramanga has kept and projected a commitment with the high quality processes in its programmes, this has been recognised by the academic community through out external peers and by the National Government, obeying to the development of the strategic axles defined by its Plan and Development integrated with the self regulation on the Institution and its undergraduate and postgraduate programmes. This article is looking for registering the self evaluation and continuous improvement processes developed by The Dentistry Faculty of Santo Tomas University Campus Bucaramanga during the Validity of High Quality Accreditation given by The National Ministry of Education by Resolution 3164, December $11^{\text {th }} 2003$. The recognition of high quality standard renewed by Resolution 517 February 6th 2008 becomes an award to the continuous improvement responding the local, regional, national and international needs.

Key word: Self-evaluation, Self-regulation, Accreditation.

Recibido para publicación: 7 de junio de 2008. Aceptado para publicación: 18 de junio de 2008.

\section{INTRODUCCIÓN}

El proceso de renovación de la acreditación de programas de acuerdo con el Consejo Nacional de Acreditación, CNA, ${ }^{2}$ implica desarrollar acciones institucionales y del programa para mantener las fortalezas y solucionar las debilidades autoevaluadas mediante un proceso participativo y transparente en el que la comunidad universitaria se hace responsable del avance frente a las recomendaciones de la evaluación externa y final de la acreditación otorgada.
La Universidad Santo Tomás expresó la voluntad de renovar la acreditación del programa de Odontología y destinó los recursos necesarios para apoyar el proceso de autoevaluación y el plan de autorregulación del programa. ${ }^{3,4}$ Los comités de gestión de la facultad: Comité de Autoevaluación y Regulación, Comité Curricular y Comité Asesor de Investigación, así como el Consejo de la Facultad participaron activamente en el proceso. La autoevaluación del programa se desarrolló mediante el modelo de 
autoevaluación para programas diseñado por el CNA con las adaptaciones necesarias a la identidad institucional y la naturaleza del programa de Odontología. 5,6

Los pares académicos designados por el Consejo Nacional de Acreditación para la evaluación externa del programa con fines de renovación de la acreditación, fueron el Dr. Alberto Arango Botero, coordinador y el Dr. Gabriel Jaime Cadavid. La evaluación final realizada por el Consejo Nacional de Acreditación recomendó a la Ministra de Educación otorgar la renovación de la acreditación mediante Resolución 717 del 6 de febrero de 2008 . $^{7}$

\section{Proyecto Educativo}

El proyecto educativo del programa de Odontología en coherencia con el proyecto institucional tomasino define el compromiso con la formación de odontólogos integrales y especialistas a través de parámetros humanistas, éticos, científicos, biotecnológicos, investigativos y sociales, como recurso humano capaz de intervenir con éxito en el proceso dinámico de la salud y la enfermedad, en el individuo, la familia y la comunidad..$^{3-8}$

El plan curricular vigente se estructura en áreas que promueven integralmente el desarrollo de capacidades y competencias profesionales a través de los procesos de: aprender a conocer, aprender a ser, aprender a hacer y aprender a desaprender y a re-aprender. ${ }^{3-8}$

En el área Social Comunitaria y Humanista, el estudiante reconoce los fenómenos sociales de acuerdo con los diversos modos explicativos del proceso salud enfermedad tanto a nivel individual como colectivo para formular objetivos y aplicar acciones que aporten a la calidad de vida y desarrollo de hábitos saludables.

El área de Básicas Biomédicas articula los conocimientos para interpretar los fenómenos biológicos de los estados de salud y enfermedad para intervenir idóneamente en la promoción de las salud, prevención de la enfermedad y recuperación de la salud.

En el área Profesional, el estudiante relaciona y fundamenta desde el punto de vista biológico, psicológico y social la promoción de la salud, la prevención y el tratamiento de las patologías bucales para el diagnóstico, tratamiento y pronóstico adecuado a través de la endodoncia, periodoncia, rehabilitación, cirugía y odontopediatría.

En el área de Investigación, el estudiante comprende las condiciones que hacen posible el co- nocimiento y la utilidad de la investigación para la resolución de problemas y la mejora de la práctica odontológica.

\section{Experiencias significativas del programa du- rante la acreditación}

Durante el tiempo de vigencia de la acreditación se registra el fortalecimiento de la cultura de la autoevaluación y el mejoramiento que ha logrado permear la vida universitaria. Las principales experiencias que impulsan la dinámica señalada son las siguientes: ${ }^{3}$

La indexación de la revista "Ustasalud Odontología" en el catálogo nacional de Colciencias, Publindex en categoría C. ${ }^{9}$

Grupos de investigación de la Facultad de Odontología con parámetros de Colciencias. ${ }^{10} \mathrm{El}$ marco de la política investigativa de la Seccional ha sido propicio para que la Facultad afiance la cultura investigativa. El grupo SIBAM, Salud Integral y Bienestar para el Adulto Mayor, el CYDUSTA, Crecimiento y Desarrollo registrados en Colciencias se encuentra en proceso final de clasificación.

La oportunidad de ser sede del XVII Encuentro de Investigación Nacional y I de la IADR. La preparación previa del evento incluyó la capacitación de los docentes para la evaluación de proyectos de investigación, la interacción con los diferentes grupos nutrió significativamente el compromiso con la investigación propiamente dicha y el desarrollo del trabajo colaborativo y cooperativo. ${ }^{10}$

La adopción del sistema de créditos académicos que impulsa el perfeccionamiento docente en torno a las metodologías de la educación superior. Los docentes y estudiantes avanzan en la apropiación del cambio de rol, se destinan esfuerzos importantes para continuar mejorando los procesos de enseñanza aprendizaje. ${ }^{8} \mathrm{El}$ seguimiento del sistema favorece el fortalecimiento de medios y mediaciones para lograr los propósitos trazados en torno a la flexibilización curricular.

El fortalecimiento del programa de asesorías de estudiantes de la Facultad y del Programa de Apoyo Académico Institucional, PAAI, han permitido disminuir la deserción estudiantil y reducir el tiempo de duración promedio para obtener el grado. ${ }^{11}$

La inversión tecnológica representada en la adquisición de la radiovisiografía en las clínicas odontológicas y de equipos de alta tecnología en laboratorios, abre un horizonte de fortalecimiento tecnológico al servicio de las comunidades y de la academia. Este hecho es considerado significativo para el desarro- 
llo de los programas de pregrado y posgrados de la Facultad al servicio de la comunidad. ${ }^{3}$

\section{AUTOEVALUACIÓN}

El proceso de autoevaluación con fines de renovación de la acreditación del programa atendió los lineamientos emitidos por el Consejo Nacional de Acreditación ${ }^{2-5}$ para renovación y las directrices de la Vicerrectoría Académica Seccional establecidos en la ruta para el seguimiento del plan de autorregulación y la realización de la autoevaluación. El modelo aplicado fue el vigente propuesto por el CNA en la Guía para Autoevaluación de Programas, 2006.

\section{Ponderación}

La ponderación o definición de la importancia relativa de características y factores fue actualizada para aplicar el nuevo modelo evaluativo. La metodología de carácter democrático se desarrollo en un taller de dos sesiones. Se conformaron ocho grupos de estudiantes y ocho grupos de docentes, cada uno de los cuales participó en la ponderación de los ocho factores y el conjunto de características. ${ }^{13}$ Un total de 16 grupos participaron en la actividad de ponderación; el grupo de docentes estuvo integrado por 16 docentes y el de estudiantes por 42. El total de participantes fue de 58 .

La tabla 1 registra el resultado de la ponderación de los factores realizada en el año $2002,{ }^{14-16}$ y la actualización en el nuevo modelo aplicado en el 2006, el cual fue el referente para la identificación de aproximación y de cumplimiento para la autoevaluación con fines de renovación de la acreditación del programa.

Tabla 1. Ponderación de factores Autoevaluación 2002 - Autoevaluación 2006

\begin{tabular}{|l|c|l|c|}
\hline \multicolumn{1}{|c|}{ PONDERACIÓN 2002 } & \multicolumn{2}{c|}{ PONDERACIÓN 2006 } & Factor \\
\hline & $\begin{array}{c}\text { Peso } \\
\text { Relativo }\end{array}$ & \multicolumn{1}{c|}{ Pelativo } \\
\hline 1. Proyecto institucional & $15 \%$ & 1. Misión y proyecto institucional & $13 \%$ \\
\hline 2. Estudiantes y profesores & $18 \%$ & 2. Estudiantes & $12 \%$ \\
\hline 3. Procesos académicos & $17 \%$ & 3. Profesores & $11 \%$ \\
\hline 4. Bienestar institucional & $11 \%$ & 4. Procesos académicos & $22 \%$ \\
\hline 5. Organización, administración y gestión. & $12 \%$ & 5.Bienestar institucional & $9 \%$ \\
\hline 6. Egresados e impacto sobre el medio. & $13 \%$ & 6. Organización, administración y gestión & $12 \%$ \\
\hline 7. Recursos fisicos y financieros & $14 \%$ & 7. Egresados e impacto sobre el medio & $10 \%$ \\
\hline & & 8. Recursos fisicos y financieros & $11 \%$ \\
\hline
\end{tabular}

\section{Metodología}

La población participante estuvo conformada por estudiantes, docentes, directivos, empleados, egresados, empleadores y usuarios del servicio. ${ }^{3}$ Se diseñaron diversos instrumentos con preguntas orientadas a identificar el nivel de acuerdo de los ítems formulados en forma positiva y para el registro individual de aspectos por mejorar. En los talleres realizados con estudiantes y docentes por semestres, se registraron los acuerdos sobre el estado de cumplimiento de las características por factor del nuevo modelo, el estado de avance sobre las recomendaciones señaladas por el CNA y las acciones de autorregulación para mantener las fortalezas, reorientar acciones o solucionar debilidades.

\section{Emisión de juicios}

A partir de la ponderación se procedió a calificar el grado de cumplimiento de cada característica, se utilizó la misma escala aplicada en la autoevaluación de $2002 .{ }^{14}$ De la combinación de la ponderación de cada característica con la calificación de su grado de cumplimiento se obtuvo la valoración de las características y del factor al que pertenecen. La tabla 2 registra el grado de cumplimiento de cada característica en la autoevaluación 2006 y su relación con el resultado de la autoevaluación realizada en el 2002. 
Tabla 2. Resultados en características de la autoevaluación 2002 y de 2006. Modelo de autoevaluación CNA 2003-2006.

\begin{tabular}{|c|c|c|c|c|c|c|}
\hline \multirow{2}{*}{\multicolumn{2}{|c|}{$\begin{array}{c}\text { FACTOR / } \\
\text { No. } \\
\text { Característica }\end{array}$}} & \multirow{2}{*}{$\begin{array}{l}\text { CARACTERÍSTICAS DEL } \\
\text { MODELO CNA } 2003 \\
\text { APLICADO PARA LA } \\
\text { AUTOEVALUCIÓN } 2006\end{array}$} & \multicolumn{2}{|c|}{ AUTOEVALUACIÓN $2002^{14}$} & \multicolumn{2}{|c|}{ AUTOEVALUACIÓN $2006^{3}$} \\
\hline & & & \multirow{2}{*}{$\begin{array}{c}\text { APROXIMACION } \\
\text { AL PESO } \\
\text { RELATIVO } \\
83.3(1)\end{array}$} & \multirow{2}{*}{$\begin{array}{c}\text { INTERPRETACIÓN } \\
\begin{array}{c}\text { Se cumple en alto } \\
\text { grado }\end{array}\end{array}$} & \multirow{2}{*}{$\begin{array}{c}\text { APROXIMACION } \\
\text { AL PESO } \\
\text { RELATIVO } \\
93.7\end{array}$} & \multirow{2}{*}{$\begin{array}{c}\text { INTERPRETACIÓN } \\
\text { Se cumple plenamente }\end{array}$} \\
\hline \multirow{4}{*}{ 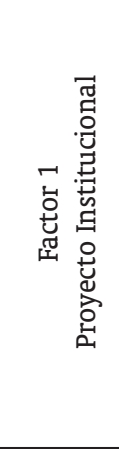 } & 1 & Misión institucional & & & & \\
\hline & 2 & Proyecto institucional & $81.8(2)$ & $\begin{array}{l}\text { Se cumple en alto } \\
\text { grado }\end{array}$ & 90 & Se cumple plenamente \\
\hline & 3 & $\begin{array}{c}\text { Proyecto educativo del } \\
\text { programa }\end{array}$ & $66.6(3)$ & $\begin{array}{c}\text { Se cumple } \\
\text { aceptablemente }\end{array}$ & 85.8 & $\begin{array}{l}\text { Se cumple en alto } \\
\text { grado }\end{array}$ \\
\hline & 4 & $\begin{array}{c}\text { Relevancia académica } \\
\text { y pertinencia social del } \\
\text { programa }\end{array}$ & No evaluada & & 82.5 & $\begin{array}{l}\text { Se cumple en alto } \\
\text { grado }\end{array}$ \\
\hline \multirow{5}{*}{ i } & 5 & Mecanismo de ingreso & $66.7(4)$ & $\begin{array}{c}\text { Se cumple } \\
\text { aceptablemente }\end{array}$ & 83.6 & $\begin{array}{l}\text { Se cumple en alto } \\
\text { grado }\end{array}$ \\
\hline & 6 & $\begin{array}{l}\text { Número y calidad de los } \\
\text { estudiantes admitidos }\end{array}$ & $81.8(5)$ & $\begin{array}{l}\text { Se cumple en alto } \\
\text { grado }\end{array}$ & 86.6 & $\begin{array}{l}\text { Se cumple en alto } \\
\text { grado }\end{array}$ \\
\hline & 7 & $\begin{array}{c}\text { Permanencia y deserción } \\
\text { estudiantil }\end{array}$ & $70(6)$ & $\begin{array}{c}\text { Se cumple } \\
\text { aceptablemente }\end{array}$ & 77 & $\begin{array}{l}\text { Se cumple en alto } \\
\text { grado }\end{array}$ \\
\hline & 8 & $\begin{array}{c}\text { Participación en } \\
\text { actividades de formación } \\
\text { integral }\end{array}$ & No evaluada & & 77.8 & $\begin{array}{l}\text { Se cumple en alto } \\
\text { grado }\end{array}$ \\
\hline & 9 & Reglamento estudiantil & $83.3(8)$ & $\begin{array}{l}\text { Se cumple en alto } \\
\text { grado }\end{array}$ & 78.4 & $\begin{array}{l}\text { Se cumple en alto } \\
\text { grado }\end{array}$ \\
\hline \multirow{8}{*}{ 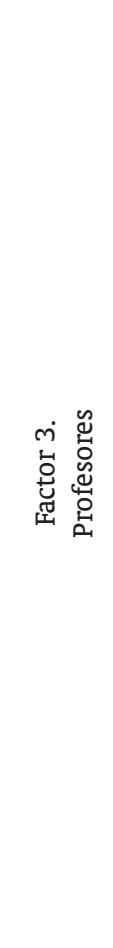 } & 10 & $\begin{array}{l}\text { Selección y vinculación de } \\
\text { profesores }\end{array}$ & $68(7)$ & $\begin{array}{c}\text { Se cumple } \\
\text { aceptablemente }\end{array}$ & 88.6 & Se cumple plenamente \\
\hline & 11 & Estatuto profesoral & $83.3(8)$ & $\begin{array}{l}\text { Se cumple en alto } \\
\text { grado }\end{array}$ & 85.7 & Se cumple plenamente \\
\hline & 12 & $\begin{array}{c}\text { Número, dedicación y } \\
\text { nivel de formación de los } \\
\text { profesores }\end{array}$ & $81.8(9)$ & $\begin{array}{l}\text { Se cumple en alto } \\
\text { grado }\end{array}$ & 81.6 & $\begin{array}{l}\text { Se cumple en alto } \\
\text { grado }\end{array}$ \\
\hline & 13 & Desarrollo profesoral & $75(17)$ & $\begin{array}{l}\text { Se cumple en alto } \\
\text { grado }\end{array}$ & 75 & $\begin{array}{l}\text { Se cumple en alto } \\
\text { grado }\end{array}$ \\
\hline & 14 & $\begin{array}{c}\text { Interacción con las } \\
\text { comunidades académicas }\end{array}$ & $83.3(18)$ & $\begin{array}{l}\text { Se cumple en alto } \\
\text { grado }\end{array}$ & 76 & $\begin{array}{l}\text { Se cumple en alto } \\
\text { grado }\end{array}$ \\
\hline & 15 & $\begin{array}{l}\text { Estímulos a la docencia, } \\
\text { investigación, extensión } \\
\text { o proyección social y a la } \\
\text { cooperación internacional }\end{array}$ & $56.2(20)$ & $\begin{array}{c}\text { Se cumple } \\
\text { insatisfactoriamente }\end{array}$ & 76.2 & $\begin{array}{l}\text { Se cumple en alto } \\
\text { grado }\end{array}$ \\
\hline & 16 & $\begin{array}{l}\text { Producción de material } \\
\text { docente }\end{array}$ & $60(33)$ & $\begin{array}{c}\text { Se cumple } \\
\text { aceptablemente }\end{array}$ & 78.8 & $\begin{array}{l}\text { Se cumple en alto } \\
\text { grado }\end{array}$ \\
\hline & 17 & $\begin{array}{l}\text { Remuneración por } \\
\text { méritos }\end{array}$ & $50(19)$ & $\begin{array}{c}\text { Se cumple } \\
\text { insatisfactoriamente }\end{array}$ & 81 & $\begin{array}{l}\text { Se cumple en alto } \\
\text { grado }\end{array}$ \\
\hline
\end{tabular}




\begin{tabular}{|c|c|c|c|c|c|c|}
\hline \multirow{14}{*}{ 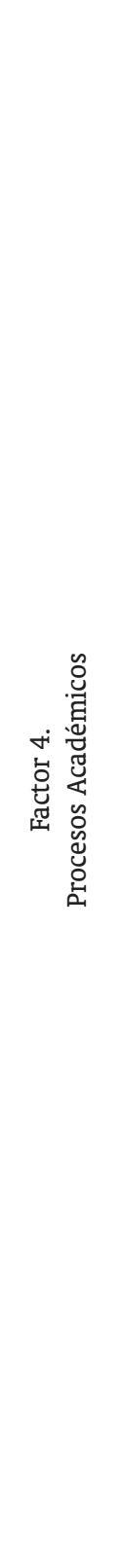 } & 18 & Integralidad del currículo & $89.3(21)$ & $\begin{array}{l}\text { Se cumple } \\
\text { plenamente }\end{array}$ & 85.2 & Se cumple plenamente \\
\hline & 19 & Flexibilidad del currículo & $56(23)$ & $\begin{array}{l}\text { Se cumple } \\
\text { insatisfactoriamente }\end{array}$ & 71 & $\begin{array}{c}\text { Se cumple } \\
\text { aceptablemente }\end{array}$ \\
\hline & 20 & Interdisciplinariedad & $72.2(26)$ & $\begin{array}{c}\text { Se cumple } \\
\text { aceptablemente }\end{array}$ & 74.7 & $\begin{array}{c}\text { Se cumple } \\
\text { aceptablemente }\end{array}$ \\
\hline & 21 & $\begin{array}{c}\text { Relaciones nacionales } \\
\text { e internacionales del } \\
\text { programa }\end{array}$ & No evaluada & & 72.1 & $\begin{array}{c}\text { Se cumple } \\
\text { aceptablemente }\end{array}$ \\
\hline & 22 & $\begin{array}{l}\text { Metodologías de } \\
\text { enseñanza y aprendizaje }\end{array}$ & $75(24)$ & $\begin{array}{l}\text { Se cumple en alto } \\
\text { grado }\end{array}$ & 78 & $\begin{array}{l}\text { Se cumple en alto } \\
\text { grado }\end{array}$ \\
\hline & 23 & $\begin{array}{c}\text { Sistema de evaluación de } \\
\text { estudiantes }\end{array}$ & $66.6(27)$ & $\begin{array}{c}\text { Se cumple } \\
\text { aceptablemente }\end{array}$ & 76 & $\begin{array}{l}\text { Se cumple en alto } \\
\text { grado }\end{array}$ \\
\hline & 24 & $\begin{array}{l}\text { Trabajos de los } \\
\text { estudiantes }\end{array}$ & $81.2(28)$ & $\begin{array}{l}\text { Se cumple en alto } \\
\text { grado }\end{array}$ & 82.5 & $\begin{array}{l}\text { Se cumple en alto } \\
\text { grado }\end{array}$ \\
\hline & 25 & $\begin{array}{c}\text { Evaluación y } \\
\text { autorregulación del } \\
\text { programa } \\
\end{array}$ & $80(29)$ & $\begin{array}{l}\text { Se cumple en alto } \\
\text { grado }\end{array}$ & 81.3 & $\begin{array}{l}\text { Se cumple en alto } \\
\text { grado }\end{array}$ \\
\hline & 26 & $\begin{array}{l}\text { Formación para la } \\
\text { investigación }\end{array}$ & $77.7(31)$ & $\begin{array}{l}\text { Se cumple en alto } \\
\text { grado }\end{array}$ & 80 & $\begin{array}{l}\text { Se cumple en alto } \\
\text { grado }\end{array}$ \\
\hline & 27 & $\begin{array}{l}\text { Compromiso con la } \\
\text { investigación }\end{array}$ & $73.3(30)$ & $\begin{array}{c}\text { Se cumple } \\
\text { aceptablemente }\end{array}$ & 80 & $\begin{array}{l}\text { Se cumple en alto } \\
\text { grado }\end{array}$ \\
\hline & 28 & $\begin{array}{l}\text { Extensión y proyección } \\
\text { social }\end{array}$ & $66.7(32)$ & $\begin{array}{c}\text { Se cumple } \\
\text { aceptablemente }\end{array}$ & 80 & $\begin{array}{l}\text { Se cumple en alto } \\
\text { grado }\end{array}$ \\
\hline & 29 & Recursos bibliográficos & $83.3(34)$ & $\begin{array}{l}\text { Se cumple en alto } \\
\text { grado }\end{array}$ & 80 & $\begin{array}{l}\text { Se cumple en alto } \\
\text { grado }\end{array}$ \\
\hline & 30 & $\begin{array}{l}\text { Recursos informáticos y } \\
\text { de comunicación }\end{array}$ & $83.3(35)$ & $\begin{array}{l}\text { Se cumple en alto } \\
\text { grado }\end{array}$ & 77.1 & $\begin{array}{l}\text { Se cumple en alto } \\
\text { grado }\end{array}$ \\
\hline & 31 & $\begin{array}{c}\text { Recursos de apoyo } \\
\text { docente }\end{array}$ & $86.6(36)$ & $\begin{array}{l}\text { Se cumple en alto } \\
\text { grado }\end{array}$ & 86.5 & $\begin{array}{l}\text { Se cumple en alto } \\
\text { grado }\end{array}$ \\
\hline 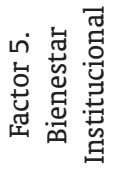 & 32 & $\begin{array}{c}\text { Políticas, programas y } \\
\text { servicios de bienestar } \\
\text { universitario }\end{array}$ & $78(40)$ & $\begin{array}{l}\text { Se cumple en alto } \\
\text { grado }\end{array}$ & 82 & $\begin{array}{l}\text { Se cumple en alto } \\
\text { grado }\end{array}$ \\
\hline :옳 & 33 & $\begin{array}{c}\text { Organización, } \\
\text { administración y gestión } \\
\text { del programa }\end{array}$ & $85.7(41)$ & $\begin{array}{l}\text { Se cumple en alto } \\
\text { grado }\end{array}$ & 82.2 & $\begin{array}{l}\text { Se cumple en alto } \\
\text { grado }\end{array}$ \\
\hline 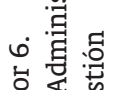 & 34 & $\begin{array}{c}\text { Sistema de comunicación } \\
\text { e información }\end{array}$ & $80(42)$ & $\begin{array}{l}\text { Se cumple en alto } \\
\text { grado }\end{array}$ & 80 & $\begin{array}{l}\text { Se cumple en alto } \\
\text { grado }\end{array}$ \\
\hline 劳: 总 & 35 & Dirección del programa & No evaluada & & 79.8 & $\begin{array}{l}\text { Se cumple en alto } \\
\text { grado }\end{array}$ \\
\hline क. & 36 & Promoción del programa & No evaluada & & 90.8 & Se cumple plenamente \\
\hline
\end{tabular}




\begin{tabular}{|c|c|c|c|c|c|c|}
\hline \multirow{3}{*}{ 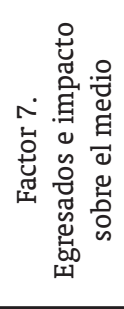 } & 37 & $\begin{array}{l}\text { Influencia del programa } \\
\text { en el medio }\end{array}$ & $80(43)$ & $\begin{array}{l}\text { Se cumple en alto } \\
\text { grado }\end{array}$ & 85.3 & $\begin{array}{l}\text { Se cumple en alto } \\
\text { grado }\end{array}$ \\
\hline & 38 & $\begin{array}{l}\text { Seguimiento de los } \\
\text { egresados }\end{array}$ & $75(46)$ & $\begin{array}{l}\text { Se cumple en alto } \\
\text { grado }\end{array}$ & 78 & $\begin{array}{l}\text { Se cumple en alto } \\
\text { grado }\end{array}$ \\
\hline & 39 & $\begin{array}{c}\text { Impacto de los egresados } \\
\text { en el medio social y } \\
\text { académico }\end{array}$ & $86.6(47)$ & $\begin{array}{l}\text { Se cumple en alto } \\
\text { grado }\end{array}$ & 80 & $\begin{array}{l}\text { Se cumple en alto } \\
\text { grado }\end{array}$ \\
\hline \multirow{3}{*}{ 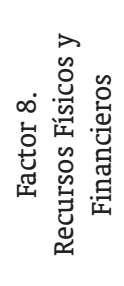 } & 40 & Recursos físicos & $93.3(48)$ & $\begin{array}{c}\text { Se cumple } \\
\text { plenamente }\end{array}$ & 90.5 & Se cumple plenamente \\
\hline & 41 & Presupuesto del programa & $80(49)$ & $\begin{array}{l}\text { Se cumple en alto } \\
\text { grado }\end{array}$ & 80 & $\begin{array}{l}\text { Se cumple en alto } \\
\text { grado }\end{array}$ \\
\hline & 42 & $\begin{array}{l}\text { Administración de } \\
\text { recursos }\end{array}$ & & & 88 & $\begin{array}{l}\text { Se cumple en alto } \\
\text { grado }\end{array}$ \\
\hline
\end{tabular}

En la tabla 3 se registra el resultado del juicio de calidad de la Autoevaluacion realizada en el 2006, ob- tenida de la relación entre el peso relativo, la aproximación identificada y el nivel de cumplimiento. ${ }^{3}$

Tabla 3. Resultados en factores de la autoevaluación 2006.

\begin{tabular}{|l|c|c|c|c|c|}
\hline \multicolumn{1}{|c|}{ FACTOR } & $\begin{array}{c}\text { PESO } \\
\text { RELATIVO }\end{array}$ & $\begin{array}{c}\text { APROX } \\
\text { AL PESO } \\
\text { RELATIVO }\end{array}$ & CUMPLIMIENTO & CATEGORÍA & INTERPRETACIÓN \\
\hline 1. Misión y proyecto institucional & 13 & 11.54 & 88.74 & B & Se cumple en alto grado \\
\hline 2. Estudiantes & 15 & 12.08 & 80.5 & B & Se cumple en alto grado \\
\hline 3. Profesores & 14 & 11.37 & 81.2 & B & Se cumple en alto grado \\
\hline 4. Procesos académicos & 22 & 12.65 & 79.07 & B & Se cumple en alto grado \\
\hline 5. Bienestar institucional & 9 & 7.38 & 82 & B & Se cumple en alto grado \\
\hline 6. Organización, administración y gestión & 12 & 9.85 & 82.0 & B & Se cumple en alto grado \\
\hline 7. Egresados e impacto sobre el medio & 10 & 8.16 & 81.55 & B & Se cumple en alto grado \\
\hline 8. Recursos físicos y financieros & 11 & 9.59 & 87.18 & B & Se cumple en alto grado \\
\hline Juicio global & 100 & 82.50 & & B & Se cumple en alto grado \\
\hline
\end{tabular}

\section{EVALUACIÓN EXTERNA}

La visita de evaluación externa se cumplió durante los días 20, 21 y 22 de septiembre de 2007. Se constituyó en un escenario propicio para el fortalecimiento de la cultura de autoevaluación en el que estudiantes, docentes, egresados y directivos en diálogo académico demostraron el sentido de pertenencia y de compromiso con el mejoramiento del programa y de la institución.

Los Pares Académicos registraron en el informe de evaluación externa las siguientes propiedades del proceso educativo del programa, por las cuales consideran que tiene alta calidad: ${ }^{15}$

- Lo histórico se mantiene vigente y cada vez se actualiza, pero se conserva la esencia del origen institucional, de manera que se mantenga en el contexto.

- El querer ser, se expresa con claridad y se conserva en la misión tanto de la Universidad como en la del programa de Odontología.
- El ser, se va formando con una institución que ha definido un propósito, un objetivo general y varios objetivos particulares, lo cual es la base para hacer un programa que incluye, con el mismo interés e importancia lo epistemológico y lo axiológico, como elementos indispensables para una formación integral de todos los actores de la práctica educativa.

- El saber, se logra con base en lo anterior, que es lo endógeno de la institución y con lo exógeno que responde a la legislación, al desarrollo de la ciencia y de la tecnología, elementos que va incorporando el currículo del programa, cada vez, al hacer los ajustes necesarios y las evaluaciones permanentes, ajustes que además de promover la eliminación de programas administrativos, agilizan el desarrollo de la formación integral.

- El saber hacer, se incrementa y garantiza con varias actividades, tales como el desarrollo docente y la asistencia personalizada a los alumnos, que son complementos importantes en lo común cu- 
rricular. Así mismo la excelente infraestructura que garantiza el desarrollo de la comunicación, de la información, del bienestar, de la ciencia y de la tecnología.

- El saber si el hacer es como debe ser o no, para juzgar la calidad de la práctica, se asegura con el desarrollo de la investigación en diferentes campos, la cual ha venido incrementando, de manera cuali-cuantitativa, el programa en sus diferentes niveles de desarrollo.

- Además de lo anteriormente planteado, que contribuye al calificativo de alta calidad, ha sido el haber logrado que algunas debilidades formuladas como recomendaciones, en el informe de evaluación externa para la primera acreditación del programa, hayan sido transformadas en aspectos positivos o fortalezas, ${ }^{15}$ entre los cuales están:

- Disminución de la deserción estudiantil.

- Introducción del tema de créditos en el plan de estudios.

- Refuerzo en investigación.

- Estímulo e incremento de las relaciones con los egresados.

\section{EVALUACIÓN FINAL}

El Consejo Nacional de Acreditación (CNA) registró las siguientes recomendaciones que hacen parte de la resolución de renovación de la acreditación del programa:

- Intensificar la asignación de tiempos, recursos y dedicación de los profesores a la actividad investigativa.

- Propender por la formación en doctorado y maestría de un mayor número de profesores.

- Consolidar los grupos de investigación existentes en la facultad y dotarlos de características que les permitan mayor visibilidad en el contexto local, regional y nacional.

- Dotar la revista Ustasalud Odontología de características que le permitan un mayor nivel en el escalafón Colciencias.

- Estimular la producción académica de los profesores y la divulgación en medios reconocidos por la comunidad académica del campo de la odontología. ${ }^{7}$

\section{AUTORREGULACIÓN}

El Plan de Autorregulación de la Facultad de Odontología expresa criterios para la evaluación y seguimiento de los procesos y proyectos que permiten identificar permanentemente el nivel de pertinencia del programa. El compromiso de directivos, do- centes, estudiantes y egresados es mantener y proyectar las fortalezas institucionales y del programa, superar las debilidades y proyectar el nivel de calidad alcanzado.

"Estamos en permanente rumbo hacia la excelencia y sobre la base de lo ya construido con el esfuerzo de todos podemos avanzar con mayor firmeza que ayer". ${ }^{16}$

\section{BIBLIOGRAFÍA}

1. Universidad Santo Tomás, Seccional Bucaramanga, Plan de Acción 2007-2010.

2. Consejo Nacional de Acreditación, CNA. Guía para la Renovación de la Acreditación de programas Académicos de pregrado. Noviembre de 2006.

3. Facultad de Odontología, Universidad Santo Tomás Seccional Bucaramanga, Informe de autoevaluación, Documento Central 2006.

4. Facultad de Odontología, Universidad Santo Tomás Seccional Bucaramanga, Informe de Autoevaluación, Anexo D Autorregulación, Vol II, 2006.

5. Consejo Nacional de Acreditación, CNA. Lineamientos para la Acreditación de programas. Bogotá, D.C. Colombia Agosto de 2003.

6. Consejo Nacional de Acreditación, CNA. Autoevaluacion con fines de acreditación de programas de pregrado. Cuarta Edición. Guía de procedimiento CNA No. 03 Bogotá Colombia, Noviembre de 2006.

7. Ministerio de Educación Nacional República de Colombia, Resolución No. 517, 6 de febrero de 2006, Acreditación voluntaria al programa de Odontología de la Universidad Santo Tomás.

8. Facultad de Odontología, Universidad Santo Tomás Seccional Bucaramanga, Informe de Autoevaluación, Anexo E Estructura curricular, Volumen III, 2006.

9. Facultad de Odontología, Universidad Santo Tomás Seccional Bucaramanga, Informe de Autoevaluación, Anexo G Revista Ustasalud Odontología, Volumen IV, 2006.

10. Facultad de Odontología, Universidad Santo Tomás Seccional Bucaramanga, Informe de Autoevaluación, Anexo F Investigación, Volumen IV, 2006.

11. Facultad de Odontología, Universidad Santo Tomás Seccional Bucaramanga, Informe de Autoevaluación, Anexo K Programa de Apoyo Académico, Voumenl IV, 2006.

12. Universidad Santo Tomás, Vicerrectoría Académica, Autoevaluación y Regulación Académica. Ruta crítica proceso de renovación de la acreditación de programas, 2004.

13. Facultad de Odontología, Universidad Santo Tomás Seccional Bucaramanga, Informe de Autoevaluación, Anexo B Ponderación, Volumen II, 2006.

14. Facultad de Odontología, Universidad Santo Tomás Seccional Bucaramanga, Informe de Autoevaluación, Documento Central, 2002.

15. Arango A, Cadavid GJ. Informe de la Evaluación Externa con fines de Renovación de la Acreditación del Programa de Pregrado de Odontología de la Universidad Santo Tomás, Seccional Bucaramanga.

16. Latorre MC. Autoevaluación y acreditación del programa de odontología de la USTA, Ustasalud Odontología 2004; 3: $7-12$. 